\title{
Non-Linear Companding Transform aided MIMO-OFDM Systems
}

\author{
K. Srinivasa Rao ${ }^{1}$, B. Prabakararao ${ }^{2}$ \\ ${ }^{I}$ G. V. P. College of Engineering for Women, Visakhapatnam, \\ ${ }^{2}$ J. N. T University, Kakinada,
}

\begin{abstract}
In this paper a new non-linear companding transform (NCT) technique based on inverse hyperbolic cosine function is proposed and implemented along with new Intercarrier interference reduction technique to improve the performance of Multi Input and Multi Output Orthogonal Frequency Division Multiplexing (MIMO-OFDM) system in terms of Peak-to-Average Power Ratio (PAPR) and Inter Carrier Interference (ICI). An effective trade-off between the PAPR and Bit Error Rate (BER) performance is achieved by introducing an inflection point and variable companding parameters in the companding function, such that it enables more design flexibility and freedom in companding form. As per theoretical study, a desired PAPR threshold can be achieved by proposed technique, since it introduces the companding distortion as small as possible with appropriate selection of the companding parameters. And new ICI cancellation scheme maintains orthogonality among subcarriers of OFDM symbol, since it decreases the Carrier to Interference Ratio (CIR). The analytical expressions regarding the achievable PAPR reduction transform gain $G$, signal attenuation factor and CIR expression are derived. Simulation results demonstrate this combination together substantially improves performance of the MIMO-OFDM system.
\end{abstract}

Keywords: MIMO-OFDM, PAPR, ICI, non-linear companding transform (NCT), CIR, high power amplifiers (HPA).

\section{Introduction}

Robust, high spectral efficiency and immunity to the interference caused by multipath fading of OFDM and very high data rate transmission of MIMO highlights the combination of MIMO-OFDM systems [1, 2]. MIMO-OFDM has been adopted in 3GPP-LTE and IEEE 802.16 (WI-MAX) standards [3]. However, this system has two major drawbacks viz., High Peak-to-Average Power Ratio (PAPR) of the transmitted signals leads to in-band distortion across subcarriers and undesired spectral growth if the linear range of higher power amplifier (HPA) is not sufficient at the OFDM transmitter [4]. Second one is Intercarrier interference among subcarriers leads to distortion in received OFDM signal as orthogonality is destroyed among subcarriers of OFDM signal.

Many different types of PAPR reduction methods have been proposed in the literature [3] -[10], e.g. Clipping and filtering, selective mapping (SLM), partial transmit sequence (PTS), block coding, active constellation extension (ACE), and companding transform techniques [11] -[18]. Among existing methods, clipping and filtering is a simple procedure to obtain the desired PAPR reduction, but it yields additional clipping noise, resulting in significant out-of-band interference (OBI) and other methods SLM, PTS require Side Information (SI) to be transmitted to recover the OFDM signal at the receiver Recently, an efficient approach called non-linear companding transform (NCT) was proposed to overcome the drawback of OBI. This technique was first introduced in [11], in which mu-law companding was implemented which efficiently outer performs the clipping. However, after implementing such logarithmic based compression on OFDM symbol, its average power increases, leads to more sensitive to the HPA. Since then, many different proposals have been made to refine NCT schemes in which two careful designs are considered to improve its overall performance. The first design focuses on optimizing the nonlinear characteristics of the companding profiles [12] -[14]. The second one is to employ the statistical distribution of the OFDM signal. To achieve more significant PAPR reduction of OFDM signal, a novel technique based on the inverse hyperbolic cosine function is proposed. In this proposal, significant PAPR reduction and an improved bit error rate (BER) performance can be achieved simultaneously, since the power distribution of the companded signal can be reallocated more reasonably while maintain the unchanged average power level. Moreover, an effective trade-off between the PAPR and BER performance can be achieved by adjusting the variable companding parameters, also it allows more flexibility and freedom in the companding form. Both theoretical analysis and simulation results confirm the effectiveness of this technique.

In addition to the high PAPR, OFDM is more sensitive to Inter Carrier Interference (ICI). It occurs due to mainly two reasons, viz. One is a mismatch in OFDM transmitter and receiver carrier frequencies and a mismatch is Doppler shift of the transmitter or receiver. Because of this effect, orthogonality among subcarrier fails, in turn distortion occurs in recovered signal and hence BER performance reduced significantly. A variety 
of ICI reduction techniques have been proposed to eliminate ICI, e.g. Carrier frequency offset (CFO) estimation and removal [19-23], a frequency domain equalization scheme [24] etc.., among existing techniques, carrier frequency estimation requires exact estimation processes which may increase the complexity of the receiver and frequency domain equalization technique includes highly complex matrix inversion, which also increases the complexity of OFDM receiver. Therefore a simple and efficient technique "new ICI cancellation scheme" [24] is selected and implemented along with proposed PAPR reduction technique for improving the performance of MIMO-OFDM systems. In the new ICI cancellation scheme, data symbols are repeated on two symmetrically located subcarriers, if ICI occurs, then the coefficients of ICI on symmetrical subcarriers are cancelled each other.

The remainder of this paper is organized as follows: section 2 reviews a typical MIMO-OFDM system using new-ICI self cancellation technique and NCT techniques for ICI and PAPR reduction. The proposed technique and corresponding theoretical analysis are derived in section 3. In section 4 , simulation results are presented and compared with existing NCT techniques. The conclusions are given in section 5 .

\section{PAPR and CIR Formulation for OFDM Signals in MIMO-OFDM.}

Fig. 1 illustrates the generic block diagram of an STBC-OFDM system with MT transmitter and MR receiver antennas with full rate Alamouti spatial coding using new ICI reduction and NCT units are implemented before and after inverse fast Fourier transform (IFFT) block in OFDM system. At the transmitter side, a serial bit stream is mapped to a symbol stream by digital modulator and converted it into parallel sub streams. Then OFDM modulation is employed in each of these sub streams by performing IFFT operation.

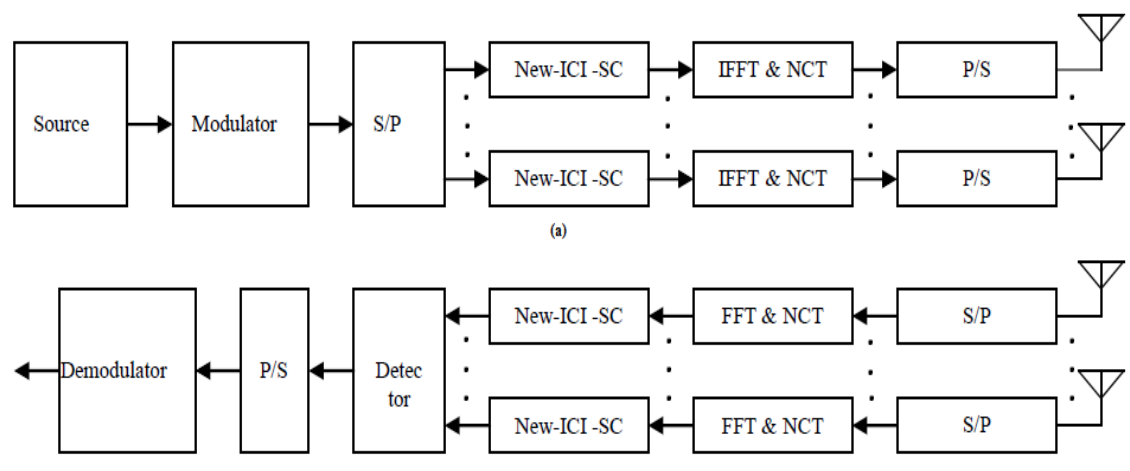

Fig. 1 Block diagram of 2 x 2 MIMO-OFDM system using new ICI-SC and NCT techniques

2 X 2 MIMO Alamouti coding can expressed as in (1)

$G=\left[\begin{array}{cc}X(2 v) & X(2 v+1) \\ -X^{*}(2 v+1) & X^{*}(2 v)\end{array}\right]$

where $v=0,1,2, \ldots, \frac{N_{c}}{2}-1$

Symbols $X(0), X(1)$ and $-X^{*}(1), X^{*}(0)$ are transmitted over antennas 1 and 2 at tones ' $\mathrm{n}$ ' and ' $\mathrm{n}+1$ ' respectively. Let $X_{P}=\left[X_{P}(0), X_{P}(1), \ldots, X_{p}(N-1)\right]^{T}$ be the frequency domain sequence from the $p^{\text {th }}$ transmit antenna to be transmitted in an OFDM symbol, where $\mathrm{N}$ is the number of subcarriers. Since Nyquist rate samples are not sufficient to represent the peaks of the continuous time signal, it is preferable to have the PAPR performance on the over-sampled time domain vector $x_{p}=\left[x_{p}(0), x_{p}(1), \ldots, x_{p}(L N-1)\right]^{T}$ is obtained after performing a LN point IFFT, where L represents the oversampling factor. Thereby the $n^{\text {th }}$ element of $x_{p}$ can be expressed as

$x_{n}=\frac{1}{\sqrt{L N}} \sum_{k=0}^{N-1} X_{k} \cdot \exp \left(\frac{j \cdot 2 \pi n k}{L N}\right), \quad n=0,1, \ldots, L N-1$

where $j=\sqrt{-1}$, and $\mathrm{n}$ is the time index. According to central limit theorem [16], $x_{n}$ can be approximated as a complex Gaussian process for a large value of $\mathrm{N}$ (e.g. $\mathrm{N} \geq 64$ ). Thus, with zero mean and a constant variance $\sigma^{2}=E\left\{\left|X_{k}\right|^{2}\right\} / 2$, the real and imaginary parts of $x_{n}$ become Gaussian distribution with probability density function (PDF) as follows

$f_{\left|x_{n}\right|}=\frac{2 x}{\sigma^{2}} \cdot \exp \left(-\frac{x^{2}}{\sigma^{2}}\right), \quad x \geq 0$ 
As a result, it is evident that the maximal amplitude of the OFDM signal may exceed its average signal amplitude. The probability distribution function (CDF) of $\left|x_{n}\right|$ is given by

$$
\begin{aligned}
& F_{\left|x_{n}\right|}(x)=\operatorname{Pr} o b\left\{\left|x_{n}\right| \leq x\right\}=\int_{0}^{x} \frac{2 y}{\sigma^{2}} \cdot \exp \left(-\frac{y^{2}}{\sigma^{2}}\right) d y \\
& =1-\exp \left(-\frac{x^{2}}{\sigma^{2}}\right), \quad x \geq 0
\end{aligned}
$$

where $\operatorname{Prob}\{\mathrm{B}\}$ is the probability of an event B.

Mathematically, the PAPR for OFDM signal is defines as

$$
P A P R_{x}=\frac{\max _{n \in[0, L N-1]}\left\{\left|x_{n}\right|^{2}\right\}}{E\left\{\left|x_{n}\right|^{2}\right\}}
$$

where E $\{$.$\} denotes the average value. It is useful to treat PAPR is a random variable and use a statistical$ description given by the complementary cumulative distribution function (CCDF) defined as the probability that exceeds a particular threshold $\mathrm{PAPR}_{0}$, i.e.

$C C D F_{x}\left(P A P R_{0}\right)=\operatorname{Pr} o b\left\{P A P R_{x}>P A P R_{0}\right\}$

By employing NCT technique, the PAPR of the original OFDM signal $\mathrm{x}_{\mathrm{n}}$ is remarkably reduced before they are converted into analog signals.

\subsection{Scheme description}

\section{Theoretical analysis of proposed scheme.}

The generic formulae of the new scheme are derived in this subsection, whose companding function is defined by an inverse hyperbolic cosine function with inflexion point. Using this method, the amplitude or power of the transmitted signal can be reallocated with reasonable and flexible manner. Moreover, it has the advantage of keeping average output power unchanged. Hence, the sensitivity of companding operation to the non-linearity of the HPA can be reduced. In OFDM system, the companded signal $y=\left[y_{0}, y_{1}, \ldots, y_{L N-1}\right]^{T}$ is given by

$y_{n}=\operatorname{Comp}\left(x_{n}\right), \quad n=0,1, \ldots, L N-1$

where $\mathrm{x}_{\mathrm{n}}$ is the original OFDM signal, and Comp(.) represents the companding function, which is strictly monotonic increasing function and only change the amplitude of $\mathrm{x}_{\mathrm{n}}$. The inverse of companding function is the de-companding function, i.e. $\operatorname{Comp}^{-1}(\mathrm{x})$. Suppose $\mathrm{cA}_{\mathrm{x}}(0 \leq \mathrm{c} \leq 1)$ is the inflexion point of Comp(x), where $A_{x}=\max _{n \in[0, L N-1]}\left\{\left|x_{n}\right|\right\}$ is the maximum input amplitude. The proposed companding function is defined as follows

$$
\operatorname{Comp}(x)=\left\{\begin{array}{c}
\operatorname{sgn}(x) \cdot \gamma \cdot \operatorname{arcosh}(k|x|),|x| \leq c A_{x} \\
\operatorname{sgn}(x) \cdot \gamma \cdot \operatorname{arcosh}\left(k c A_{x}\right),|x|>c A_{x}
\end{array}\right.
$$

where $\operatorname{sgn}($.$) is the sign function, and the inverse hyperbolic cosine function \operatorname{arcosh}($.$) is given by$

$$
\operatorname{arcosh}(x)=\ln \left(x+\sqrt{x^{2}-1}\right)
$$

The average power can be adjusted by the parameter ' $\gamma$ ' and the degree of companding specified by parameter ' $k$ '. Before and after companding operation, the average power of the signal is same according to the proposed method.

$$
E\left\{\left|y_{n}\right|^{2}\right\}=E\left\{\left|x_{n}\right|^{2}\right\}=\int_{0}^{\alpha} x^{2} f_{\left|x_{n}\right|}(x) d x
$$

Clearly, the parameter ' $\gamma$ ' can be determined, when ' $\mathrm{k}$ ' is chosen. At the companded signal can be recovered by the corresponding de-companding function.

$$
\operatorname{Comp}^{-1}(x)=\operatorname{sgn}(x) \cdot \frac{1}{k} \cdot \cosh \left(\frac{|x|}{\gamma}\right)
$$

where $\cosh (x)=\frac{e^{x}-e^{-x}}{2}$ is the hyperbolic cosine function.

Further by making approximate substitution, the CDF of companded signal may be obtained as

$$
\begin{aligned}
& F_{\left|y_{n}\right|}(x)=\operatorname{Prob}\left\{\left|y_{n}\right| \leq x\right\}=\operatorname{Prob}\left\{\operatorname{comp}\left(x_{n}\right) \leq x\right\} \\
& =\left\{\begin{array}{c}
0, x<0 \\
1-\exp \left(-\frac{\cosh ^{2}(x / \gamma)}{k^{2} \sigma^{2}}\right), 0 \leq x \leq \gamma \cdot \operatorname{ar} \cosh \left(k c A_{x}\right) \\
1, x \geq \gamma \cdot \operatorname{ar} \cosh \left(k c A_{x}\right)
\end{array}\right.
\end{aligned}
$$


The proposed method is suitable for real time applications as it used in practical OFDM systems through the use of pre-computed look-up tables.

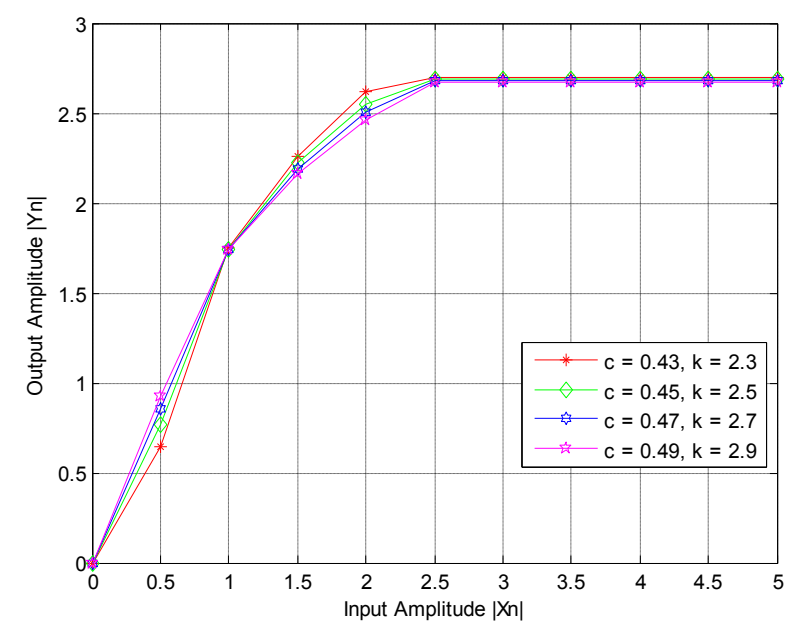

Fig. 2 Transform profiles of the proposed companding function with various parameters.

Fig. 2 depicts the transform profile of the companding function in (8) with various parameters. As shown in the figure, the proposed scheme compresses large amplitude signals while partially improves the small amplitude signals simultaneously. Moreover, this method does not increase the average power as in the case of $\mu$-law companding method. Thus, this technique effectively reduces the PAPR by reducing the peak signals and also provides immunity of small signals from the channel noise.

In the following subsections, its theoretical performance in terms of achievable PAPR reduction and impact of companding distortion on BER performance was investigated.

\subsection{Achievable PAPR reduction.}

As per the PAPR definition in (5), the maximum PAPR of the companded signal can be derived as follows.

$$
\operatorname{PAPR}_{y}=\frac{\max _{n \in[0, L N-1]}\left\{\left|y_{n}\right|^{2}\right\}}{E\left\{\left|y_{n}\right|^{2}\right\}}=\frac{\gamma^{2} \cdot \operatorname{arcosh}\left(k c A_{x}\right)}{\sigma^{2}}
$$

In order to compare the PAPR values before and after the companding operation, a transform gain $G$ [12], is defined as the ration of the PAPR of original signal to that of companded signal, i.e.

$$
G(d B)=10 \log _{10} \frac{P A P R_{x}}{P A P R_{y}}=20 \log _{10} \frac{A_{x}}{\gamma \cdot \operatorname{arcosh}\left(k c A_{x}\right)}
$$

The theoretical results of the attainable PAPR reduction and transform gain G, obtained using (13) and (14), respectively. As can be seen, the proposed technique can effectively limit the final PAPR in the range [3 $\mathrm{dB}, 9.5 \mathrm{~dB}$ ] by adjusting the variable companding parameters. In addition, the plots also illustrate that the more $\mathrm{PAPR}$ reduction can be obtained along with $\mathrm{c}$ and $\mathrm{k}$ increasing.

Furthermore, the CCDF of the achievable PAPR using proposed technique can be formulated as $C C D F_{y}\left(P A P R_{0}\right)=\operatorname{Prob}\left\{P A P R_{y}>P A P R_{0}\right\}$

$$
=C C D F_{x}\left(\frac{A_{x}^{2}}{\gamma^{2} \cdot a \cosh ^{2}\left(k c A_{x}\right)} P A P R_{0}\right)
$$




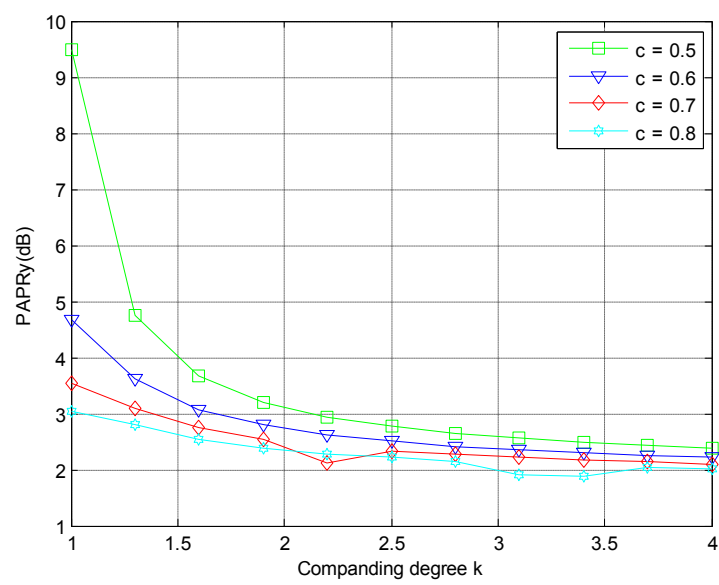

(a)

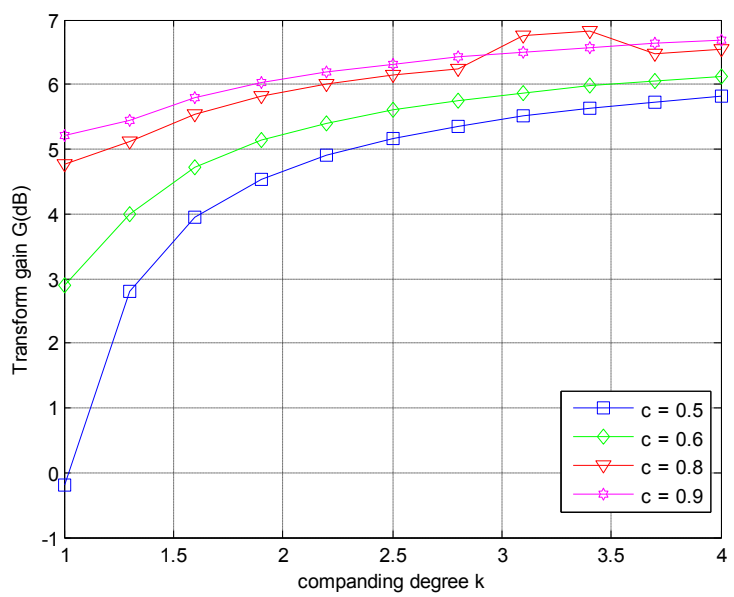

(b)

Fig. 3 Theoretical results of PAPR and $G$ with various companding parameters. a) PAPR of the companded signals, b) transform gain $G$.

\subsection{Distortion due to Companding transforms}

Extra non-linear process is introduced to the transmitted signal by NCT companding operation, so it is clear that when PAPR reduced, corresponding BER performance may be degraded. As discussed in [12], due to the disadvantages of non-linear distortion, NCT should be designed carefully such that the amount of clipped signals as little as possible. Hence, it may be advisable to minimize the impact of companding distortion on BER as small as possible. The companded signal can be represented as the sum of a useful attenuated input replica and companding noise as per the extension of Bussgang theorem [26].

$y_{n}=\alpha . x_{n}+b_{n}, n=0,1, \ldots, L N-1$

where $\alpha$ is an attenuation factor and can be calculated as

$\alpha=\frac{E\left\{y_{n} \cdot x_{n}^{*}\right\}}{E\left\{x_{n} \cdot x_{n}^{*}\right\}}=\frac{1}{\sigma^{2}} \int_{0}^{\alpha} x \cdot \operatorname{Comp}(x) \cdot f_{\left|x_{n}\right|}(x) d x$

Considering an additive white Gaussian noise (AWGN) channel, the received signal $r_{n}$ can be expressed as $r_{n}=y_{n}+w_{n}$, where $w_{n}$ is channel noise. After the de-companding operation, the recovered signal $x_{n}^{\prime}$ can be expressed as [14]

$x_{n}^{\prime}=\frac{y_{n}+x_{n}}{\alpha}-\frac{b_{n}}{\alpha}=x_{n}+\frac{w_{n}}{\alpha}$

From eq. (18), it is clear that channel noise $w_{n}$ enlarges to $\frac{w_{n}}{\alpha}$ after de-companding operation. To minimize the signal distortion $\alpha$ must approach to ' 1 ' enough. Substituting $\operatorname{Comp}(\mathrm{x})$ and $f_{\left|x_{n}\right|}(x)$ in (17), the attenuation factor of this technique is obtained as 
$\alpha=\frac{2 \gamma}{\sigma^{4}} \int_{0}^{c A_{x}} x^{2} \exp \left(-\frac{x^{2}}{\sigma^{2}}\right) \operatorname{arcosh}(k x) d x+\frac{2 \gamma}{\sigma^{4}} \operatorname{arcosh}\left(k c A_{x}\right) \int_{c A_{x}}^{A x} x^{2} \exp \left(-\frac{x^{2}}{\sigma^{2}}\right) d x$

(19)

From the simulation results, approaches to ' 1 ' for the values of $\mathrm{c}=0.8, \mathrm{k}=1.15$. It can be observed that, by choosing the optimal companding parameters the proposed scheme approached the desired PAPR level. Also from the Fig. 3, it is noteworthy that, even though the PAPR reduction and BER performance affect each other, but an effective trade-off between them can be achieved to satisfy various requirements.

\section{CIR of ICI reduction technique}

ICI reduction technique mitigates the interference among sub carriers. Various simple ICI cancellation techniques are ICI Self Cancellation (SC), new ICI-SC, ICI-complex conjugate, and new ICI - complex conjugate technique. In conventional ICI-SC technique, the data symbols are repeated on two or more adjacent subcarriers i.e. Complex data symbols and are to be transmitted over and subcarriers respectively from the transmitter. At the receiver the difference between the symbols received on the two adjacent subcarriers will be taken, this process is ICI cancellation demodulation. But Conventional ICI-SC cannot achieve much frequency diversity on two adjacent subcarriers since they experience same channel frequency response. Therefore a modified ICI self cancellation called new ICI-SC technique is used to improve the CIR performance over frequency selective fading channel. In this technique data symbols are repeated on two symmetrically located subcarriers using polynomial. $X(N-1)=-X(0), X(N-2)=-X(1), \ldots, X(N-K)=-X(K)$.

The expression of discrete time domain OFDM signal using above expression can be represented as

$$
\begin{aligned}
& x(n)=\frac{1}{\sqrt{N}} \sum_{k=0}^{N / 2-1} X_{k}\left\{\exp \left(\frac{j 2 \pi k n}{N}\right)-\exp \left(\frac{j 2 \pi(N-1-K) n}{N}\right)\right\} \\
& =\frac{2 j}{\sqrt{N}} \exp \left(\frac{-j \pi n}{N}\right) \sum_{k=0}^{\frac{N}{2}-1} X_{k} \sin \left(\frac{j 2 \pi\left(k+\frac{1}{2}\right) n}{N}\right)
\end{aligned}
$$

The expression of CIR performance for new ICI self cancellation techniques is given as

$$
C I R_{N S C}=\frac{|2 x(0)|^{2}}{\sum_{l=1}^{N / 2-1}|x(l)-x(N-1-l)-x(l-N+1)+x(-l)|^{2}}
$$

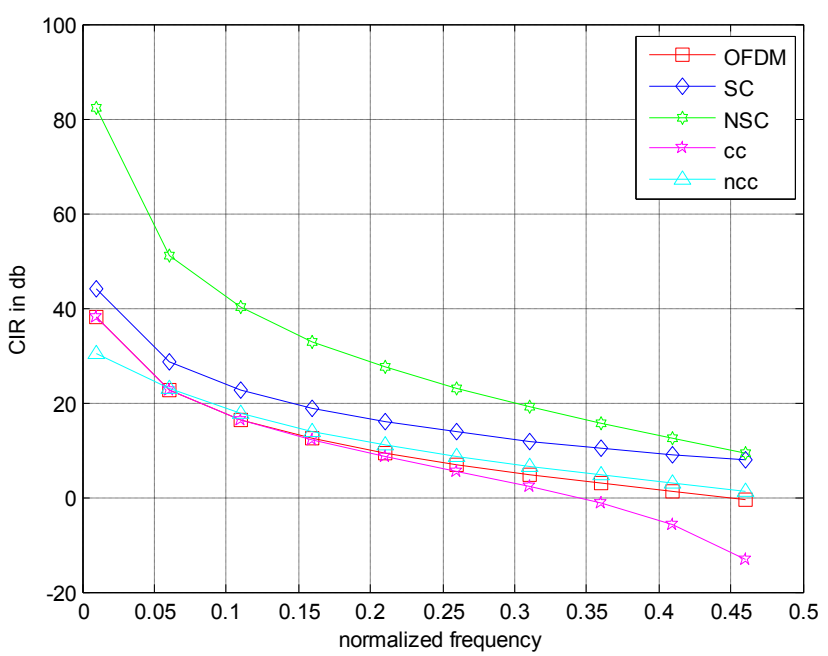

Fig. 4 CIR performances of various ICI cancellation schemes

As can be seen from Fig. 4 it is evident that the CIR performance of new ICI-SC scheme is superior to other techniques. Hence this method is employed to improve the system performance along with proposed PAPR reduction technique.

\section{Simulation results}

Alamouti STBC 2 X 2 MIMO-OFDM with $\mathrm{N}=512$ subcarriers using quaternary phase shift keying (QPSK), and 16-QAM is simulated. For the performance comparison purpose, two classic NCT methods, including - law [11], and NCT1 [18] were also simulated. 


\subsection{Performance in PAPR reduction}

1000 OFDM symbols have been generated in order to observe the PAPR statistics. Fig. 5 represents the plots the CCDF of the PAPR for the original OFDM signal and companded signals using three different NCT methods. The PAPR of the original OFDM signal with QPSK is $11.05 \mathrm{~dB}$. As seen from the figure, at clip rate $10^{-3}$ the OFDM system using proposed technique can improve the PAPR by $10.66 \mathrm{~dB}$. Particularly this technique with $\mathrm{c}=0.45$ and $\mathrm{k}=2.5$ is surpasses $\mu$ - law, NCT1 by $6.41 \mathrm{~dB}$, and $5.01 \mathrm{~dB}$ respectively.

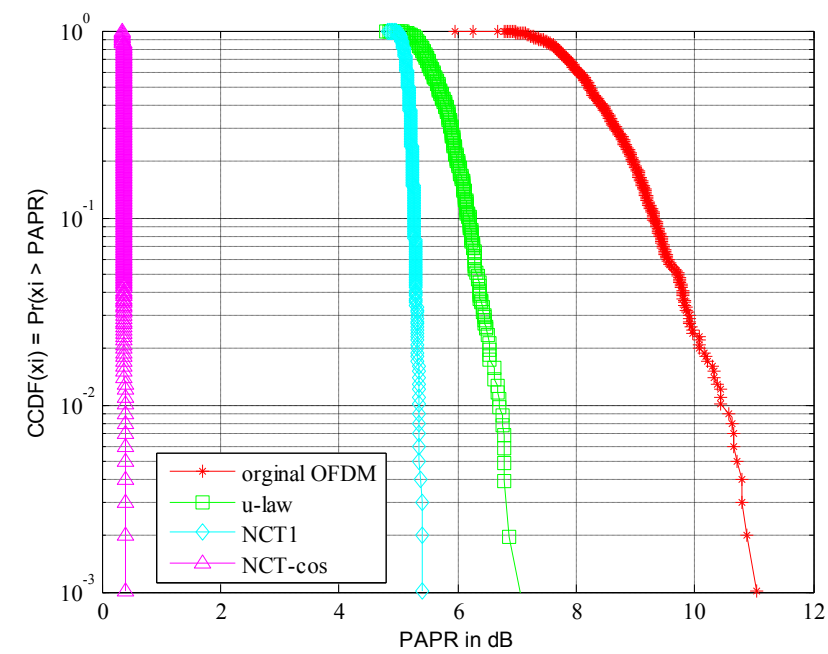

Fig. 5 CCDF comparison among original OFDM signal and the companded signal using different NCT techniques (QPSK modulation).

\subsection{BER performance.}

Fig. 6 and Fig. 7 present the BER performance Vs Eb/No curves employing different methods over an AWGN channel with QPSK and 16QAM, respectively. As a reference, the curve of ideal performance bound also given. As it apparent, the BER performance of NCT degraded, while it significantly reduces the PAPR. All the BER curves of the companded signals are located at the right of the ideal bound. As can be seen in Fig. 6 to reach a BER level of 10-4, for example, the required $\mathrm{Eb} / \mathrm{No}$ for the proposed technique with $\mathrm{c}=0.45$ and $\mathrm{k}=1.6$ is $12 \mathrm{~dB}$, which is about $1.8 \mathrm{~dB}$ to $4 \mathrm{~dB}$ superior to others. Moreover, it is noteworthy that the BER performance in QPSK case is only about $0.4 \mathrm{~dB}$ worse than the ideal bound. As expected, since the power of transmitting signal has been reallocated more reasonably, the impact of companding distortion on BER performance can be effectively reduced.

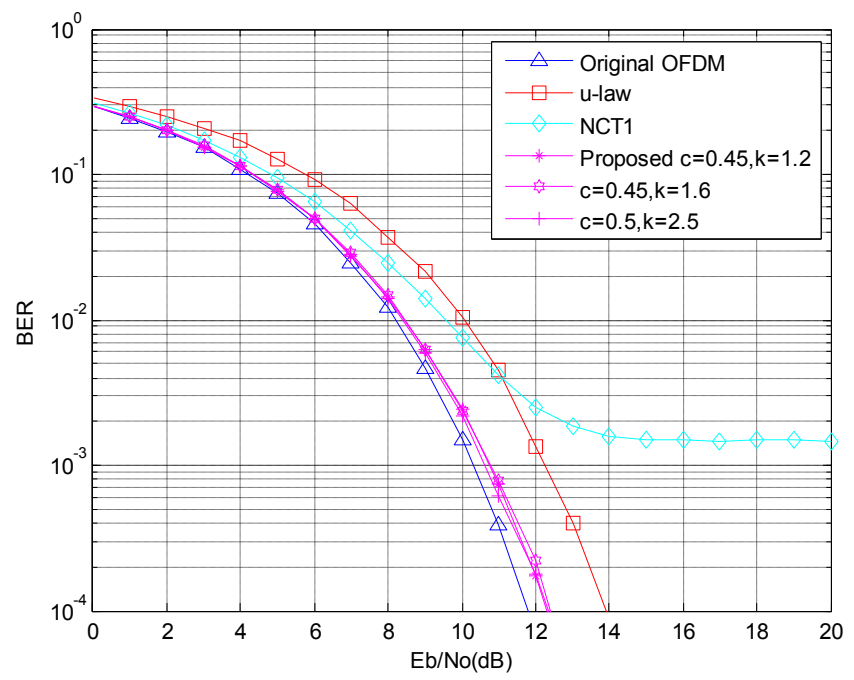

Fig. 6 BER comparison between the original signal and the companded signal employing different methods over AWGN channel (QPSK). 


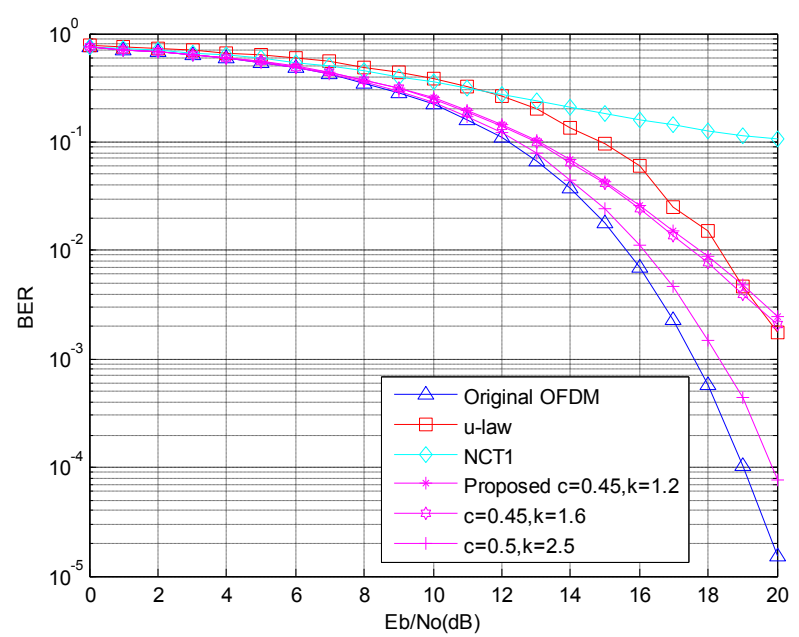

Fig. 7 BER comparison between the original signal and the companded signal employing different methods over AWGN channel (16QAM).

\section{Conclusion}

In a properly designed STBC MIMO-OFDM system, PAPR reduction, ICI cancellation and moderate BER performance are three critical challenges. In this paper a novel NCT technique employing the inverse hyperbolic cosine function has been proposed and evaluated along with new ICI cancellation technique. In the proposed NCT technique the power distribution of the companded signal can be reallocated more reasonably by compressing the large signals along with partially enhancing the small amplitudes simultaneously and in the new ICI-SC technique data symbols are repeated on two symmetrically located subcarriers so that ICI is cancelled. It has been proved that, with appropriate selection of the variable companding parameters and inflexion point in the companding function, a significant PAPR reduction can be achieved with less compounding distortion. Moreover, an effective trade off among the PAPR and BER performance can also be offered to satisfy the various design parameters. Simulation results demonstrated that the NCT technique can achieve better overall performance than existing NCT techniques in terms of the PAPR reduction, and BER performance.

\section{Acknowledgments}

The authors sincerely thanked Prof. G.T Rao, E.C.E Department, G.V.P College of Engineering (Autonomous) for his immense support and valuable suggestions.

\section{References}

[1] Y. Wu and W. Y. Zou, “Orthogonal frequency division multiplexing: A multi-carrier modulation scheme,” IEEE Trans. Consumer Electron., vol. 41, no. 3, pp. 392-399, Aug. 1995.

[2] M Sandell, J Coon, Near-optimal training sequences for MIMO OFDM systems with nulled subcarriers. in Proc. IEEE Global Telecommunications Conference GLOBECOM '05, vol. 4, 2243-2249, (2005).

[3] B Rihawi, Y Louet, S Zabre, PAPR reduction scheme with SOCP for MIMO-OFDM. in Proc. Int. Conf. Wireless Communications, Networking and Mobile Computing WiCom 2007, 271-274, (2007).

[4] B Rihawi, Y Louet, S Zabre, PAPR reduction scheme with SOCP for MIMO-OFDM. in Proc. Int. Conf. Wireless Communications, Networking and Mobile Computing WiCom 2007, 271-274, (2007).

[5] T. Hwang, C. Yang, G. Wu, S. Li, and G. Y. Lee, “OFDM and its wireless application: A survey,” IEEE Trans. Veh. Technol., vol. 58, no. 4, pp. 1673-1694, May 2009.

[6] L. Wang and C. Tellambura, "A simplified clipping and filtering technique for PAR reduction in OFDM Systems," IEEE Signal Process. Lett., vol. 12, no. 6, pp. 453-456, June 2005.

[7] J. Yang, L. Chen, Q. Liu, and D. Chen, "A modified selected mapping technique to reduce the peak-to-average power ratio of OFDM signals," IEEE Trans. Consumer Electron., vol. 53, no. 3, pp. 846-851, Aug. 2007.

[8] P. Varahram, W. F. Al-Azzo, and B. M. Ali, "A low complexity partial transmit sequence scheme by use of dummy signals for PAPR reduction in OFDM systems,” IEEE Trans. Consumer Electron., vol. 56, no. 4, pp. 2416-2420, Nov. 2010.

[9] K. D. Choe, S. C. Kim, and S. K. Park, "Pre-scrambling method for PAPR reduction in OFDM communication systems," IEEE Trans. Consumer Electron., vol. 50, no. 4, pp. 1044-1048, Nov. 2004.

[10] S. Krongold and D. L. Jones, "PAR reduction in OFDM via active constellation extension," IEEE Trans. Broadcast., vol. 49, no. 3 , pp. 258-268, Sep. 2003

[11] X. B. Wang, T. T. Tjhung, and C. S. Ng, "Reduction of peak-to-average power ratio of OFDM system using a companding technique," IEEE Trans. Broadcast., vol. 45, no. 3, pp. 303-307, Sep. 1999.

[12] X. Huang, J. Lu, J. Zheng, J. Chuang, and J. Gu, "Reduction of peak to average power ratio of OFDM signals with companding transform," IEE Elec. Lett., vol. 37, pp. 506-507, Apr. 2001.

[13] X. Huang, J. Lu, J. Zheng, K. B. Letaief, and J. Gu, "Companding transform for reduction in peak-to-average power ratio of OFDM signals," IEEE Trans. Wireless Commun., vol. 3, no. 6, pp. 2030-2039, Nov. 2004. 
[14] G. Yang, Y. Zhou, and S. Qian, "Using hyperbolic tangent sigmoid transfer function for companding transform in OFDM system," International Symposium on ISCIT 2007, pp.87-90, Oct. 2007.

[15] J. Hou, J. Ge, D. Zhai, and J. Li, "Peak-to-average power ratio reduction of OFDM signals with nonlinear companding scheme," IEEE Trans. Broadcast., vol. 56, no. 2, pp. 258-262, Jun. 2010.

[16] S.S. Jeng, and J.M. Chen, "Efficient PAPR reduction in OFDM systems based on a companding technique with trapezium distribution,” IEEE Trans. Broadcast., vol. 57, no. 2, pp. 291-298, Jun. 2011.

[17] Yuan Jiang, "New Companding Transform for PAPR Reduction in OFDM," IEEE Commun. Lett., vol. 14, no. 4, pp. 282-284, Apr. 2010 .

[18] Yong Wang, Jianhua Ge, Lihua Wang, Jing Li, "Reduction of PAPR of OFDM Signals Using Nonlinear Companding Transform", Springer Science, Business Media, LLC. 2012.

[19] van de Beek, J. J., Sandell, M., \& Borjesson, P. O. (1997). ML estimation of time and frequency offset in OFDM systems. IEEE Transactions on Signal Processing, 45(7), 1800-1805.

[20] Tureli, U., Kivanc, D., \& Liu, H. (2001). Experimental and analytical studies on a high-resolution OFDM carrier frequency offset estimator. IEEE Transactions on Vehicular Technology, 50(2), 629-643.

[21] Fernandez-Getino Garcia, M. J., Edfors, O., \& Paez-Borrallo, J. M. (2001). Frequency offset correction for coherent OFDM in wireless systems. IEEE Transactions on Consumer Electronics, 47(1), 187-193.

[22] Luise, M., Marselli, M., \& Reggiannini, R. (2002). Low-complexity blind carrier frequency recovery for OFDM signals over frequency-selective radio channels. IEEE Transactions on Communications, 50(7), 1182-1188.

[23] Ahn, J.,\&Lee, H. S. (1993). Frequency domain equalization of OFDM signals over frequency nonselective Rayleigh fading channels. IEEE Electronics Letters, 29(16), 1476-1477.

[24] Wang, C. L., \& Huang, Y. C. (2010). Inter Carrier interference cancellation using general phase rotated conjugate transmission for OFDM systems. IEEE Transactions on Communications, 58(3), 812-819.

[25] P. Banelli, "Theoretical analysis and performance of OFDM signals in nonlinear fading channels," IEEE Trans. Wireless Commun., vol. 2, no. 2, pp. 284-293, Mar. 2003.

\section{Biographical notes:}

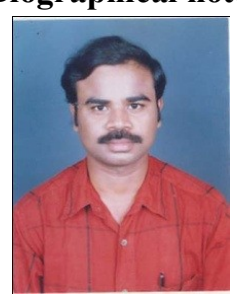

Mr. K. Srinivasa Rao received B. Tech degree in Electronics and Communication Engineering from Nagarjuna University, Guntur and M. Tech degree in I\&CS from J.N.T University College of Engineering. Kakinada, presently he is working as a Sr. Assistant Professor in department of Electronics and Communication Engineering, G.V.P. College of Engineering for Women, Visakhapatnam. His research interests include Wireless Communications and Signal processing. He is currently perusing Ph.D. from J.N.T.U. Kakinada and is a Life Member of IETE.

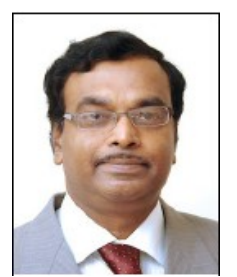

Dr.B.Prabhakara Rao received B.Tech degree in Electronics and Communications Engineering. M.Tech degree in Electronics and Communication Systems from S. V. University, Tirupati in 1979, 1981 respectively and received the Ph.D from IISc, Bangalore in 1995. Dr.B.Prabhakara Rao has more than 32 years of experience in teaching and Research. He has produced $17 \mathrm{PhDs}$ and presently 20 scholars are perusing their Ph.D. program under his guidance. He held different positions in his career like Head of the Department, Vice Principal, in JNTU College of Engineering etc., and currently he is the Rector of the JNT University. He has published more than 175 technical papers in the reputed National and International journals and conferences. His research interests are coding theory, Information theory and Signal processing with applications to wireless communications. 